\title{
De-Noising via Wavelet Transforms Using Steerable Filters
}

\author{
Andrew F. Lainet and Chun-Ming Chang $\ddagger$ \\ †Computer \& Information Sciences and Engineering \\ †Electrical and Computer Engineering \\ University of Florida \\ Gainesville, FL 32611
}

\begin{abstract}
Feature extraction remains an important part of low-level vision. Traditional oriented filters have been effective tools to identify features, such as lines and edges. Steerable filters, which can be adjusted at arbitrary orientation, have made decisions of feature orientations more precise. Combined with a pyramid structure of a multiscale representation, these filters can provide a reliable and efficient tool for image analysis.

This paper takes advantage of multiscale steerable filters in the context of de-noising. First a set of novel filters are designed, that decompose the frequency plane into distinct directional bands. Next, we identify the dominant direction and strength at each point of an image from quadrature pairs of steerable filters. A nonlinear threshold function is then applied to the filtered coefficients to suppress noise. The denoised image is restored from coefficients modified at each level of transform space. We demonstrate the benefits of multiscale steerable filters for de-noising and show that it can greatly reduce noise while preserving image features. Two examples are presented to verify the efficacy of the technique.
\end{abstract}

Keywords - Multiscale steerable filters, de-noising, basis functions, oriented energy, nonlinear thresholding.

\section{INTRODUCTION}

By definition, "noise" is any unwanted disturbance in a signal. When we obtain an image, it may be contaminated by noise from various sources. Some important features of an image will be degraded by noise, and we may hardly recognize edge locations, orientations or textures in an image. Because of such local ambiguities, a noisy image may reduce the quality of any subsequent processing. Therefore, de-noising is an essential component of an early visual processing system.

There have been many noise reduction methods discussed in the literature. The simplest noise reduction technique is equal-weighted averaging over a neighborhood (mean filtering). Averaging produces an effect similar to that produced by low-pass filtering. Therefore, reducing the effects of random noise results in blurring an image. The method of median filtering is similar to mean filtering in that it uses the local median instead of a local mean, and exhibits better performance for edge-perservation and noise reduction for speckle noise.

More recently, de-noising based on wavelet transformations have been proposed. Mallat et al. [9], [10] used the local maxima of the wavelet transform modulus to analyze image singularities. The algorithm removed all maxima whose amplitude increased on average when the scale decreased, or which did not propagate to larger scales. A "denoised" signal was then reconstructed with an alternative projection algorithm. Donoho and Johnstone [3], [4] proposed a three-step method for recovery of a signal. Their method is based on a theoretically motivated nonlinear shrinkage of wavelet coefficients. Mallat and Zhang [11] introduce a matching pursuit algorithm that decomposed a signal into a linear expansion of waveforms that belong to a redundant dictionary of functions. With a dictionary of Gabor functions, a matching pursuit defined an adaptive time-frequency transform.

Freeman and Adelson [6] proposed the concept of steerable filters and applied it to several problems. With a set of "basis filters", one can adaptively steer a filter along any orientation. Hilbert transform pairs were constructed to find a local "oriented energy" measure. Noise was then reduced by performing a soft threshold function [14] on the pyramid of multiscale coefficients.

Each visual neuron can be characterized psychophysically by its receptive field (RF). Much psychophysical evidence [2], [7] shows that the RF cell is sentive to multiple, parallel bandpass neural images. Therefore, it is useful to decompose an image into a number of lowpass and bandpass subimages. Oriented linear or piecewise linear patterns are commom phenomena in nature and contain rich sources of image information. In order to detect these features, we applied the same filter to calculate filter responses at various orientations. Multiscale steerable filters were designed in quadrature pairs at several scales to allow adaptive capture of magnitudes as well as directions of features embedded in an image.

\section{BaCkground}

\section{a. Problem formulation}

Let a noisy image be given by

$$
f(x, y)=i(x, y)+n(x, y)
$$

where $i(x, y)$ is an original image and $n(x, y)$ is noise component. It is assumed that the noise is additive, stationary, and has zero mean. We chose white Gaussian noise in our model. The purpose of de-noising is to restore an image 
that differs as little as posible from its original. Thus, we hope to preserve the features of an image while smoothing noise.

\section{b. A theorem for steerable filters}

A function $f(x, y)$ is called "steerable" if it can be expressed as a linear combination of rotated versions of itself. The fundamental idea of steerable filters is to apply "basis filters" which correspond to a fixed set of orientations and interpolate between each discrete response. Thus, one must decide the number of "basis filters" and the corresponding interpolation functions. As defined in [6] the steering constraint is formulated as

$$
f^{\theta}(x, y)=\sum_{i=1}^{M} k_{i}(\theta) f^{\theta_{i}}(x, y),
$$

where $\mathrm{M}$ is the number of basis functions required to steer a function $f^{\theta_{i}}(x, y)$.

Hereafter, it will be more convenient to work in polar coordinates $r=\sqrt{x^{2}+y^{2}}$ and $\phi=\arg (x, y)$. Let $f$ be any function that can be expressed as a Fourier series in polar angle $\phi$ :

$$
f(r, \phi)=\sum_{n=-N}^{N} a_{n}(r) e^{j n \phi},
$$

where $j=\sqrt{-1}$.

The theorem below was posed by [6] and is included for clarity of discussion.

Theorem 1: The steering condition (2) holds for function $f$ expanded in the form of (3) if and only if the interpolations $k_{i}(\theta)$ are solutions of

$$
\left(\begin{array}{c}
1 \\
e^{j \theta} \\
\vdots \\
e^{j N \theta}
\end{array}\right)=\left(\begin{array}{cccc}
1 & 1 & \cdots & 1 \\
e^{j \theta_{1}} & e^{j \theta_{2}} & \cdots & e^{j \theta_{M}} \\
\vdots & \vdots & \ddots & \vdots \\
e^{j N \theta_{1}} & e^{j N \theta_{2}} & \cdots & e^{j N \theta_{M}}
\end{array}\right)\left(\begin{array}{c}
k_{1}(\theta) \\
k_{2}(\theta) \\
\vdots \\
k_{M}(\theta)
\end{array}\right)
$$

Then $f^{\theta}(r, \phi)$ may be expressed as

$$
f^{\theta}(r, \phi)=\sum_{i=1}^{M} k_{i}(\theta) g_{i}(r, \phi),
$$

where $g_{i}(r, \phi)$ can be any set of functions.

\section{Design of Steerable Filters}

In this section we describe the procedures we employed to design our steerable filters. A function is steerable if it is bandlimited in angular frequency and there are sufficient basis functions, where each basis function is generated from dilations and rotations of a single kernel. In order to steer the filters, we generate first the basis filters. Each of these filters were designed using a polar separable frequency strategy, by separating the radial and angular parts of the bandpass filters

$$
G(\rho, \theta)=G(\rho) G(\theta)
$$

where $\rho$ is the radial frequency and $\theta$ the angular frequency.

The frequency plane was decomposed into three parts: lowpass, bandpass, and highpass partitions. Then we subsample and decompose the lowpass portions into bandpass and lowpass subportions recursively. The filters are chosen such that each single stage system (including lowpass and bandpass parts) behaves like an identity operation, and its response is lowpass (equal to $L_{0}(\omega)$ ). In addition, Simoncelli et al. [14] described constraints for the design of the radial filters:

1. Lowpass filter $L_{1}$.

Since there is a subsampling operation after $L_{1}$, in order to avoid aliasing, the lowpass filter $L_{1}$ should have strictly zero response above $\omega=\frac{\pi}{2}$.

2. Lowpass filter $L_{0}$.

It should cover the frequency bound of $L_{1}$. We let the response equal 1 for $\omega$ between 0 and $\frac{\pi}{2}$ and zero at $\omega=\pi$.

3. Bandpass filter $B$.

The constraint for the design of bandpass filter $B(\omega)$ was

$$
\left|L_{0}(\omega)\right|^{2}=|B(\omega)|^{2}+\left|L_{1}(\omega)\right|^{2}\left|L_{0}(2 \omega)\right|^{2} .
$$

4. Highpass filter $H$.

This is obtained simply by substracting the response of $L_{0}$ from unity.

It is easier to first design the filters in one-dimension in the frequency domain and then convert them to twodimensions. We used a seven-tap lowpass filter as $L_{1}(\omega)$ and generate $L_{0}(\omega)$ using the Park-McClellan algorithm [8]. Both meet constraints 1 and 2. Filter $B(\omega)$ was obtained from Equation (6). Next, each one-dimensional filter was converted into a nearly circularly symmetric two-dimensional filter, using the frequency transformation method described in [8]. The bandpass filter is shown in Fig. 1(a).

Having selected a radial frequency band, we divided the band into several oriented subbands by multiplying an angular frequency component. As in [14], $G(\theta)=j \cos ^{3}(\theta)$ was used as the angular frequency response, expressed as

$$
\cos ^{3}(\theta)=\frac{1}{4} \cos (3 \theta)+\frac{3}{4} \cos (\theta) \text {. }
$$

From (4) the minimum number of basis functions required to steer a filter is exactly four. The bandpass filter was multiplied by angular frequency $\cos ^{3}\left(\theta-\theta_{i}\right)$ to obtain the basis functions. Four basis functions are shown in Fig. 1(b), (c), (d), and (e). The interpolation functions were determined by solving (4), yielding

$$
k_{i}(\theta)=\frac{1}{4}\left[2 \cos \left(\theta-\theta_{i}\right)+2 \cos \left(3\left(\theta-\theta_{i}\right)\right)\right]
$$

where $\theta_{i}=(i-1) \pi / 4, i \in\{1, \ldots, 4\}$.

In order to compute the local energy function, we need to find the basis functions of the quadrature pair of $G(\theta)$ (i.e. they are the Hilbert transforms of each other). The Hilbert transform is related to the Fourier expansion of the image 
in that it has the same amplitude spectrum and a phase shift of $\frac{\pi}{2}$. The Hilbert transform $G^{H}(\theta)$ of $G(\theta)=j \cos ^{3}(\theta)$ is $\left|\cos ^{3}(\theta)\right|$. Following [14], this was approximated by the first three terms of the Fourier expansion of $G^{H}(\theta)$ :

$$
\left|\cos ^{3}(\theta)\right| \approx 0.4244+0.5093 \cos (2 \theta)+0.0727 \cos (4 \theta) .
$$

This needs five basis functions. The interpolation functions were

$$
k_{l}(\theta)=\frac{1}{5}\left[1+2 \cos \left(2\left(\theta-\theta_{l}\right)\right)+2 \cos \left(4\left(\theta-\theta_{l}\right)\right)\right],
$$

where $\theta_{l}=(l-1) \pi / 5, l \in\{1, \ldots, 5\}$.

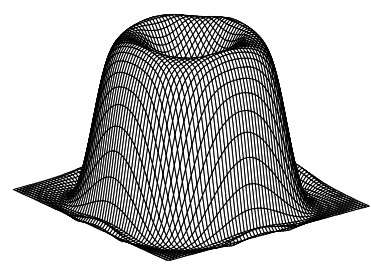

(a)

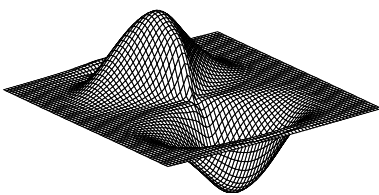

(b)

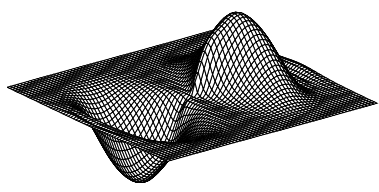

(d)

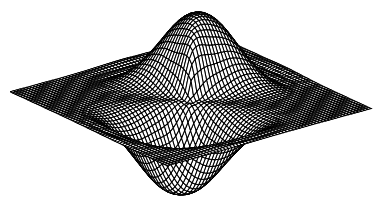

(c)

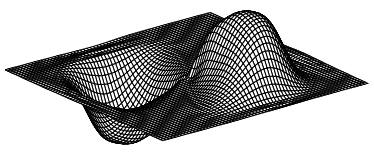

(e)
Fig. 1. (a) Two-dimensional frequency response of bandpass filter. (b)-(e) Frequency responses of four basis functions for $G$.

Having computed the basis functions, we construct the steerable functions for $G(\theta)$, as shown in Fig. 2, and its quadrature pair $G^{H}(\theta)$. In order to find its local perceptual significance, we used an "oriented energy" measure. Local "oriented energy" $E(\theta)$ [12] was defined as the sum of the squared responses of quadrature pair filters. Let the outputs of $G(\theta)$ and $G^{H}(\theta)$ be $I G(\theta)$ and $I G^{H}(\theta)$, respectively. Thus,

$$
\begin{aligned}
E(\theta)= & {[I G(\theta)]^{2}+\left[I G^{H}(\theta)\right]^{2} } \\
= & {\left[k_{1}(\theta) g_{1}+k_{2}(\theta) g_{2}+k_{3}(\theta) g_{3}+k_{4}(\theta) g_{4}\right]^{2} } \\
& +\left[k_{1}^{H}(\theta) g_{1}^{H}+k_{2}^{H}(\theta) g_{2}^{H}+k_{3}^{H}(\theta) g_{3}^{H}\right. \\
& \left.+k_{4}^{H}(\theta) g_{4}^{H}+k_{5}^{H}(\theta) g_{5}^{H}\right]^{2}
\end{aligned}
$$

where $k_{i}$ and $g_{i}(i=1, \ldots, 4)$ are interpolation functions and the outputs of the basis functions of $G(\theta)$, respectively, and $k_{l}^{H}$ and $g_{l}^{H}(l=1, \ldots, 5)$ are interpolation functions and the outputs of the basis functions of $G^{H}(\theta)$, respectively.
Following [14], equation (12) is simplified to a Fourier series in angular form using the substitutions of the trigonometric identity:

$E(\theta)=a_{0}+a_{1} \cos (2 \theta)+a_{2} \sin (2 \theta)+[$ higher order terms $\ldots]$.

The lowest frequency terms were used to approximate the dominant directions A and their associated magnitudes M.

$$
\begin{aligned}
A & =\arctan \left[a_{1}, a_{2}\right] / 2 . \\
M & =\sqrt{a_{1}^{2}+a_{2}^{2}} .
\end{aligned}
$$

A and $\mathrm{M}$ were then used to modify the pyramid coefficients of outputs of basis functions, by a nonlinear threshold function,

$$
\begin{aligned}
c_{i j}^{M O D} & =0.9 \frac{c_{i j}}{1+e^{\left.-\left(\sqrt{m_{i j}}-T\right) / 32\right)}} \quad, \text { if } \quad \sqrt{m_{i j}}-T \geq 0 . \\
& =1.1 \frac{c_{i j}}{1+e^{-\left(\sqrt{m_{i j}}-T\right)}} \quad, \text { if } \quad \sqrt{m_{i j}}-T<0 .
\end{aligned}
$$

such that a coefficient was retained if its local energy was larger and attenuated if smaller, where $c_{i j}$ is the coefficient at the dominant orientation, $m_{i j}$ is the magnitude along that orientation, and threshold T can be adjusted for distinct subbands. Thus we may reconstruct an image using the modified coefficients $c_{i j}^{M O D}$.

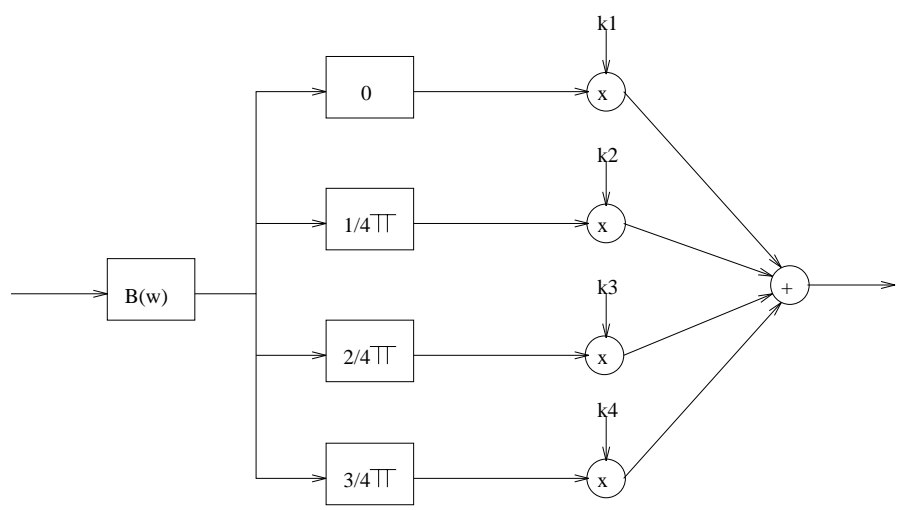

Fig. 2. Block diagram of steerable filter.

\section{Experimental Results}

Next, we shall provide two examples to demonstrate the de-noising performance using multiscale steerable filters. The image is of size $(256 \times 256)$. The first example was the familiar "Lena" test image. The original noise-free image is shown in Fig. 4(b). We added white gaussian noise and obtained a noisy image shown in Fig. 4(c). Then we decomposed the image using multiscale steerable filters (up to three scales). The pyramidal representations of coefficients are shown in Fig. 3. Fig. 4(a) shows its lowpass result at scale $=3$. The reconstructed image is shown in Fig. 4(d). Compared with the original noise-free image in Fig. 4(b), we preserved the features while eliminating the noise! The SNR of the original image is $12.6 \mathrm{~dB}$. The denoised one is $20.8 \mathrm{~dB}$ ! 
The second example is a frame from a functional MRI sequence. Fig. 5(a) shows the original image. Noise existed throughout the image and artifacts appeared on the surface of the object. After de-noising, the surrounding noise disappeared and the borders of cranial tissues appeared smoother.

\section{Conclusions and Discussions}

In this paper we applied the properties of steerable filters [6] and their design for the application of de-noising. Using oriented information to detect the features of an image appears to be a promising approach. Steerable filters which can be rotated at arbitrary orientations can more reliably find visual cues in an image. "Oriented energy" clearly helped us to discriminate features from noise. The pyramid structure of multiscale steerable filters made the computation efficient and the algorithm easy to implement. Experimental results were shown demonstrating the efficacy of de-noising.

The filters used in this paper were based on a design proposed by Freeman and Adelson [6]. However, we remove noise by employing a nonlinear thresholding operation to the transform coefficients. In addition, we used an interpolation approach for the filter design that enabled us to avoid search in the multi-dimensional space (scale space) of filter coefficients. Since the filters were non-separable, we implemented them in the frequency domain to avoid discrete two-dimensional convolution operations.

These techniques may also be applied in other applications such as stereo matching, texture analysis, motion perception, shape-from-shading analysis, etc. [1], [6], [14]. In addition, the method is extendable to three-dimensional analysis for volumetric spatial data and temporal image sequences.

Acknowledgement: Sponsored in part by National Science Foundation research grant No. IRI-9111375.

\section{REFERENCES}

[1] Adelson, E. H. and Bergen, J. R., "Saptiotemporal energy models for the perception of motion," J. Opt. Soc. Am. A, vol. 2, no. 2, Feb. 1985.

[2] Blakemore, C. and Campbell, F. W., "On the existence of neurons in human visual system selectively sensitive to the orientation and size of retinal images," $J$. Physiology London 203, 1969, $237-260$.

[3] Donoho, D. L., "De-noising via soft-thresholding," Tech. Rept., Statistics, Stanford, 1992.

[4] Donoho, D. L. and Johnstone, I. M., "Ideal spatial adaptation via wavelet shrinkage," Tech. Rept., Statistics, Stanford, 1992.

[5] Farrokhnia, F. and Jain, A. K., "A multi-channel filtering approach to texture segmentation," in IEEE comput. Soc. Conf. Comput. Vision and Pattern Recogn., Maui, 1991, pp. 364-370.

[6] Freeman, W. T. and Adelson, E. H., "The design and use of steerable filters," IEEE Trans. Pattern Anal. Machine Intell. vol. 13, pp. 891-906, 1991.

[7] Graham, N. and Nachmias, J., "Detection of grating patterns containing two spatial frequencies: A comparision of singlechannel and multiple-channel models," Vision Res. 11, 1971, 251-259.

[8] Lim, J., Two-Dimensional Signal and Image Processing, Englewood Cliffs, NJ: Prentice-Hall, 1990.
[9] Mallat, S. and Hwang, W. L., "Singularity detection and processing with wavelets," IEEE Trans. on Info. Theory, vol. 38, no. 2, March 1992.

[10] Mallat, S. and Zhang, S. "Characterization of signals from multiscale edges," IEEE Trans. Pattern Anal. and Mach. Intell., vol. 14, no. 7, July 1992.

[11] Mallat, S. and Zhang, Z., "Matching pursuits with timefrequency dictionaries," IEEE Trans. Info. Theory, vol. 41, no. 12, Dec. 1993.

[12] Morrone, M. C. and Burr, D. C., "Feature detection in human vision: A phase-dependent energy model," Proc. R. Soc. London $B$, vol. 235, pp. 221-245, 1988.

[13] Morrone, M. C. and Owens, R. A., "Feature detection from local energy," Patt. Recog. Lett., vol. 6, pp.303-313, 1987.

[14] Simoncelli, E. P., Freeman, W. T., Adelson, E. H., and Heeger, D. J., "Shiftable multiscale transforms," IEEE Trans. on Info. Theory, vol. 38, no. 2, March 1992.
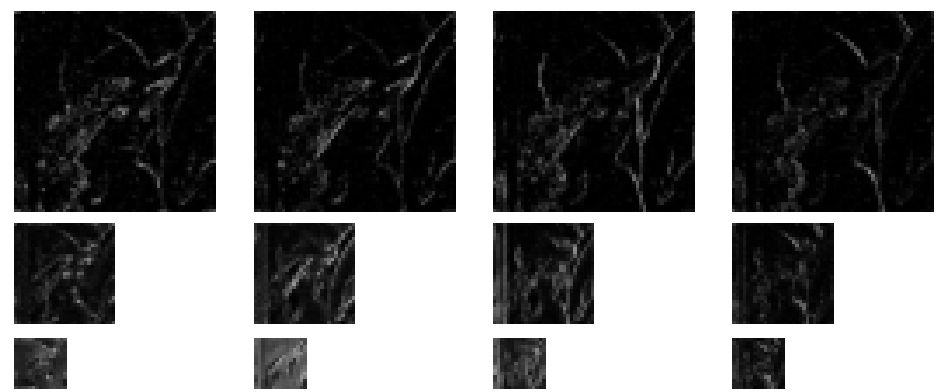

Fig. 3. Steerable bandpass coefficients of "Lena" (3 levels).

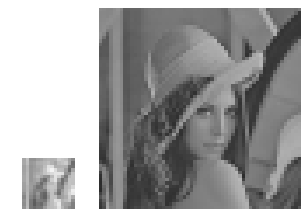

(a)

(b)

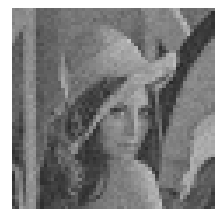

(c)

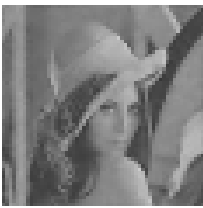

(d)
Fig. 4. Steerable image transform of "Lena". (a) Lowpass filtered and downsampled image. (b) Original image. (c) Noisy image. (d) Restored image.

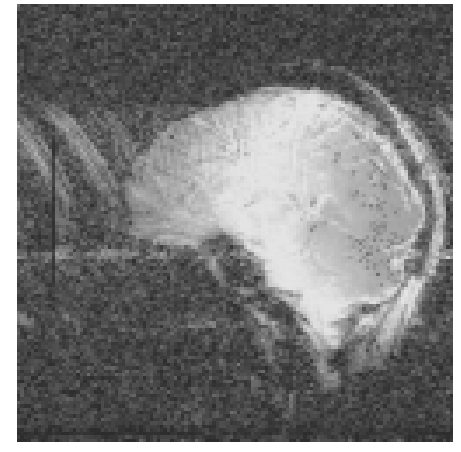

(a)

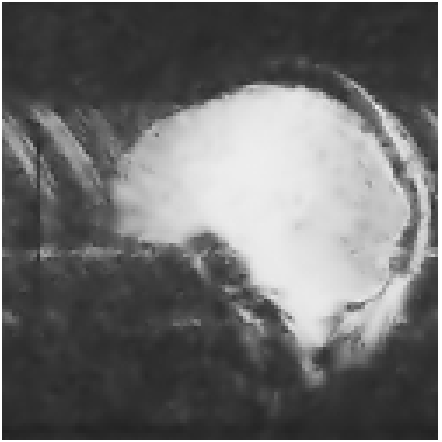

(b)
Fig. 5. Steerable image transform of MRI image. (a) Noisy image. (b) Restored image. 